\title{
Evaluation of Fear and Peritraumatic Distress during COVID-19 Pandemic in Brazil
}

\author{
Alberto Abad1*, Juliana Almeida da Silva2,3, Lucas Emmanuel Pedro de Paiva Teixeira4, \\ Mayra Antonelli-Ponti ${ }^{5}$, Sandra Bastos 6 , Cláudia Helena Cerqueira Mármora ${ }^{7}$,

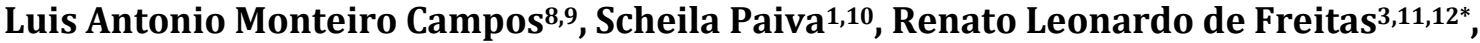 \\ José Aparecido da Silva ${ }^{13}$
}

\author{
${ }^{1}$ Psychology Graduate Program, Federal University of Juiz de Fora (UFJF), Juiz de Fora, Brazil \\ ${ }^{2}$ Laboratory of Neuroanatomy and Neuropsychobiology, Department of Pharmacology, Ribeirão Preto Medical School of the \\ University of São Paulo (FMRP-USP), Ribeirão Preto, Brazil \\ ${ }^{3}$ Behavioural Neurosciences Institute (INeC), Ribeirão Preto, Brazil \\ ${ }^{4}$ Institute of Motricity Sciences, Federal University of Alfenas (UNIFAL-MG), Alfenas, Brazil \\ ${ }^{5}$ Laboratory of Studies and Research in Social Economy, University of São Paulo at Ribeirão Preto, Ribeirão Preto, Brazil \\ ${ }^{6}$ Otorhinolaryngology Institute (ISBO), São Paulo, Brazil \\ ${ }^{7}$ Department of the Old, Adult and Maternal-Infant, School of Physical Therapy, Graduate Program in Psychology, Federal \\ University of Juiz de Fora (UFJF), Juiz de Fora, Brazil \\ ${ }^{8}$ Department of Psychology, Pontifical Catholic University of Rio de Janeiro, Rio de Janeiro, Brazil \\ ${ }^{9}$ Master's Program in Psychology, Catholic University of Petrópolis, Petrópolis, Brazil \\ ${ }^{10}$ Speech Therapy of Federal University of Sergipe (UFS), Lagarto, Brazil \\ ${ }^{11}$ Biomedical Sciences Institute, Federal University of Alfenas (UNIFAL), Minas Gerais, Brazil \\ ${ }^{12}$ Laboratory of Neurosciences of Pain \& Emotions and Multi-User Centre of Neuroelectrophysiology, Department of Surgery and \\ Anatomy, Ribeirão Preto Medical School of the University of São Paulo, Ribeirão Preto, Brazil \\ ${ }^{13}$ Laboratory of Psychophysics, Perception, Psychometrics, and Pain, University of São Paulo at Ribeirão Preto, \\ Ribeirão Preto, Brazil \\ Email: *alberto.abad@ich.ufjf.br, *defreitas.rl@gmail.com
}

How to cite this paper: Abad, A., da Silva, J.A., de Paiva Teixeira, L.E.P., AntonelliPonti, M., Bastos, S., Mármora, C.H.C., Campos, L.A.M., Paiva, S., de Freitas, R.L. and da Silva, J.A. (2020) Evaluation of Fear and Peritraumatic Distress during COVID-19 Pandemic in Brazil. Advances in Infectious Diseases, 10, 184-194.

https://doi.org/10.4236/aid.2020.103019

Received: August 5, 2020

Accepted: September 11, 2020

Published: September 14, 2020

\begin{abstract}
COVID-19 pandemic continues to spread exponentially worldwide, especially in America. At the end of August 2020, Brazil is one of the most affected countries with more than three and a half million cases and up to 114,250 deaths. This study aims to assess the fear and peri-traumatic stress during the COVID-19 pandemics in Brazil, to enhance infection control methods, appropriate interventions, and public health policies. A cross-sectional survey has been conducted from April $12^{\text {th }}$ to $18^{\text {th }}$ using the Peri-Traumatic Distress Scale (CPDI) and the Fear Scale (FCV-19S) aiming to measure the peri-traumatic stress and fear as psychological reactions during the COVID-19 pandemic. For that purpose, an online spreadsheet was used to send the questionnaire and scales to a sample of 1844 participants as a collecting information tool. Significant positive relationship $(r=0.660, p<0.001)$ and internal
\end{abstract}


Copyright $\odot 2020$ by author(s) and Scientific Research Publishing Inc. This work is licensed under the Creative Commons Attribution International License (CC BY 4.0).

http://creativecommons.org/licenses/by/4.0/

Open Access consistencies on CPDI and FCV-195 scales (Cronbach Alfa scores 0.90 and 0.88 respectively) were shown. Results highlight significant gender differences as in both scales women's mean scores are higher showing that it is paramount that women's voices were represented in policy spaces as socially constructed gender roles place them in a strategic position to enhance multi-level interventions (primary and secondary effects of COVID-19), equitable policies, and new approaches to control the pandemic.

\section{Keywords}

Mental Health, Fear, Peri-Traumatic Stress, COVID-19, Pandemic, Brazil

\section{Introduction}

The coronavirus disease pandemic (COVID-19) emerged in Wuhan, China, in late 2019; by the start of 2020, it had spread to a dozen countries. Since then, the number of cases has continued to escalate exponentially worldwide. Nowadays, Brazil is one of the most affected countries, as the disease has spread to all five regions of the country - at the start of the present study (20 August 2020), there were 3,456,652 confirmed COVID-19 cases, including 111,100 deaths.

On a bioecological approach, previous research on pandemics has revealed a profound and wide range of psychosocial impacts on people during outbreaks of the infection. On a microsystem level, people are likely to experience fear of falling sick or dying themselves, feelings of helplessness, and stigma [1], health threats to oneself and loved ones, and higher chances of being afflicted by mood swings, depression, irritability, anxiety, fear, anger, insomnia, changes in appetite or subjective well-being [2].

On a mesosystem level, pandemics are further associated with severe disruptions of routines, separation from family and friends, school closure, shortages of food and medicine, wage loss, and social isolation (due to quarantine or other social distancing programs). Additionally, with fear of infection as the health care system could not cope with the COVID-19 pandemic [3] [4]. Also, on a macrosystem level, the impact on cultural, political, and socioeconomic factors should be considered, as COVID-19 pandemics has influenced education, unemployment, and quality of work. No one can predict how things will evolve in the coming months, nor when a return to some semblance of "normal" activity might resume [5].

People from different countries experience various levels of stress, fear, or anguish. As an example, fear in China during the pandemic differs from that in Iran, Italy, and Spain, indicating the need to study mental health predictors in specific countries during the COVID-19 to effectively identify, track, and assist those people most susceptible to mental health problems [1] [6] [7] [8].

Consequently, and as a result of a collaboration involving a group of Brazilian and foreign researchers interested in the effects of the impact and the varied 
psychological states caused by the rapid spread of COVID-19 around the world, was created the research project "Physical, Psychological, and Cognitive reactions to COVID-19", approved by the Research Ethics Committee (process number: 4.128.627). The project is divided into ten modules, each one aiming to assess, or measure, some particular dimension of a psychological state derived from the COVID-19 pandemics: psychological reactions to pandemics; psychological vulnerability factors; social isolation, the role of the media and the dissemination of coronavirus-19; effective ways to deal with psychological problems; and, the implications for public health policies, including appropriate interventions for risk communication.

Besides contributing to Brazilian literature about COVID-19 pandemics, this study aims to assess the fear and peri-traumatic stress during the COVID-19 contagion in Brazil, to enhance infection control methods, appropriate interventions, and public health policies.

\section{Methods}

As the first module of the research project entitled Physical, Psychological, and Cognitive reactions to COVID-19, we conducted a cross-sectional survey from April $12^{\text {th }}$ to $18^{\text {th }}$ aiming to measure the peri-traumatic stress and fear as psychological reactions during the COVID-19 pandemics. For that purpose, we chose the COVID-19 Fear scale (FCV-19S) and Peri-Traumatic Distress Index (CPDI) designed by a team of specialists, and based on international studies and the literature on pandemics. As a collecting information tool, we used an online spreadsheet (Google Forms) to send the questionnaire and scale to the participants (via social media and email) composed as a convenience sample.

Before answering the questionnaire, candidates read and accepted the Participant Consent Form that explained the objective and nature of the study and showed they could refuse to answer any question and withdraw at any time from the research. Originally, 1875 participants were reached, but researchers had to exclude 31 incomplete questionnaires-possibly because of internet slow-down access during the questionnaire fill-out-making the final sample of 1844 people.

Participants first answered a socio-demographic survey that included specific questions about chronic disease prevalence and social isolation levels during the COVID-19 pandemic. Then, we assessed distress by the Peri-Traumatic Distress Index (CPDI), designed as a self-report questionnaire that measures depression, anxiety, avoidance, compulsive behaviour, specific phobias, cognitive change, physical symptoms, and loss of social functioning [8].

The twenty-four questions were presented in a Likert format in five categories of responses (never, occasionally, sometimes, often, most of the time). According to the authors, scores range from 0 to 100 indicating mild to moderate distress (between 28 and 51) and severe distress ( $\geq 52)$. CPDI content has been validated by Psychiatrists from the Shanghai Mental Health Center considering its Cronbach's alpha $0.95(\mathrm{p}<0.001)$ [8]. 
We measured fear with the COVID-19 Fear scale (FCV-19S) presented in a Likert format in five categories of responses (strongly disagree, disagree, neither agree nor disagree, agree, strongly agree). It consists of a seven-item unidimensional scale with robust psychometric properties (Cronbach's alpha internal consistency 0.82 ) reliable and valid in assessing and relieving fears of COVID-19 among individuals [9]. Scores range from 7 to 35 indicating levels of fear: normal (7 - 16); mild to moderate (17 - 26); and severe (27 - 35).

The authors authorized us to use the FCV-19S and CPDI scales in the research. We translated and adapted them to the Brazilian Portuguese language. We used descriptive statistics as data analysis method: CPDI and FCV-19S average scores, male and female frequency ranges, the coefficient correlation of the scales (Pearson's r) and Cronbach Alpha estimates for each of the factors of the respective scales.

\section{Results and Discussion}

We first assessed the characteristics of the participants (gender, age, marital status, number of children, education level, job status, and social isolation levels during COVID-19 pandemic) as shown in Table 1. Out of the sample, 643 participants (34.9\%) declared chronic disease prevalence, standing out suffering, or psychological disorder indicators (16.1\%). This is relevant because the COVID-19 pandemic outbreak not only hurts physically but also psychologically as it disrupts lives, causes public panic, mental health distress [10], pathological anxiety, post-traumatic stress, and depression [11].

From a COVID-19 control strategy perspective, it is also highlighted the hypertension levels (10.4\%) since non-communicable diseases (hypertension, diabetes, cardiovascular and chronic obstructive pulmonary illnesses) are correlated with the need for intensive care units [12] in a delicate political, economic, and social context that reflects the importance of flattening the curve of infections due to the fragility of the health systems and high contagion rates [2].

Based on Table 1, it is possible to build a rough profile of the participants: single $(48.7 \%)$ woman $(79.8 \%)$, with an average of 36.2 years of age, with up to one child $(75.7 \%)$, with a college degree (complete or incomplete $89.7 \%$ ), working $(69.5 \%)$ with health insurance $(69.4 \%)$ and in social isolation during the pandemic $(87.0 \%)$. The profile is psychologically significant because, although men and women are similar in many ways, it matches the biological, behavioural, and cognitive differences between genders that influence the health care approach in terms of manifestation, epidemiology, and pathophysiology widespread diseases [13].

As gender dimensions of the pandemic are both physical and socially constructed, affecting the sexes differently [14], women are perceived as being more aware of their need for healthcare, more adherent to counselling and treatment, and likely, seeking healthcare more often than men [15] that explains the higher number of female answers of this and other surveys [16]. 
Table 1. Descriptions of the participants $(n=1844)$.

\begin{tabular}{|c|c|c|}
\hline Variable & Count or mean & Percentage \\
\hline \multicolumn{3}{|l|}{ Gender } \\
\hline Female & 1471 & $79.8 \%$ \\
\hline Male & 368 & $20.0 \%$ \\
\hline Other & 5 & $0.2 \%$ \\
\hline \multicolumn{3}{|l|}{ Age (years) } \\
\hline Mean & 36.2 & \\
\hline Median & 35 & \\
\hline Mode & 22 & \\
\hline \multicolumn{3}{|l|}{ Marital Status } \\
\hline Single & 898 & $48.7 \%$ \\
\hline Married & 772 & $41.9 \%$ \\
\hline Others & 174 & $9.4 \%$ \\
\hline \multicolumn{3}{|l|}{ Number of Children } \\
\hline 0 & 1036 & $56.2 \%$ \\
\hline 1 & 360 & $19.5 \%$ \\
\hline 2 & 331 & $18.0 \%$ \\
\hline 3 & 95 & $5.2 \%$ \\
\hline More than 3 & 22 & $1.2 \%$ \\
\hline \multicolumn{3}{|l|}{ Education level } \\
\hline Secondary school or below & 4 & $0.25 \%$ \\
\hline Secondary school (complete) & 5 & $0.25 \%$ \\
\hline High school (incomplete) & 24 & $1.3 \%$ \\
\hline High School (complete) & 157 & $8.5 \%$ \\
\hline College degree (incomplete) & 396 & $21.5 \%$ \\
\hline College degree (complete) & 1258 & $68.2 \%$ \\
\hline \multicolumn{3}{|l|}{ Chronic disease } \\
\hline Total people & 643 & $34.9 \%$ \\
\hline Heart disease & 37 & $2.0 \%$ \\
\hline Hypertension & 192 & $10.4 \%$ \\
\hline Diabetes & 71 & $3.8 \%$ \\
\hline Low acuity or loss of vision & 136 & $7.4 \%$ \\
\hline Low acuity or hearing loss & 32 & $1.7 \%$ \\
\hline Terminal illness & 1 & $0.05 \%$ \\
\hline Tuberculosis & 0 & $0 \%$ \\
\hline Suffering or indicators of psychological disorder & 298 & $16.1 \%$ \\
\hline \multicolumn{3}{|l|}{ Job status } \\
\hline Working & 1281 & $69.5 \%$ \\
\hline Not working & 563 & $30.5 \%$ \\
\hline
\end{tabular}




\begin{tabular}{ccc}
\hline Health Insurance & & \\
Yes & 1279 & $69.4 \%$ \\
No & 565 & $30.6 \%$ \\
Social isolation & & \\
Yes & 1605 & $87.0 \%$ \\
No & 239 & $13.0 \%$ \\
\hline
\end{tabular}

${ }^{\star}$ Total answers (643) representing $34.9 \%$ of the sample.

Furthermore, since health services availability, governance structures, and emergency responders interactions, all have gender dimensions [14] and considering that gender roles and stigma affect adherence to counselling and treatment [15] it is possible to infer that women could be of utmost importance to control the spread of the coronavirus infection, and consequently, improving global health security [17]. In that sense, it is of utmost importance to include women's voices and knowledge in decision making, preparedness, and response to the pandemic, as there is an insufficient women's representation in global COVID-19 policy spaces [17].

Table 2 shows gender frequency scores of the Peritraumatic Distress Scale (CPDI) and the Fear Scale (FCV-19S). In both scales, women's mean scores are higher; women's CPDI frequency scores are predominantly on the mild and severe distress frequency levels ( $47.3 \%$ and $27.2 \%)$ while men rely on normal and mild levels (41.6\% and 48.4\%). Similarly, most women's FCV-19S frequency scores are at a mild level (44.5\%), while men's scores are at a normal level (68.8\%). Still, women's scores are higher $(15.4 \%)$ than men $(4.8 \%)$ at a severe level.

Results indicated that female gender was associated with increased anxiety, depression, and stress. This finding is in line with the results of previous studies that have consistently found an association between female gender and increased psychological distress [1] [7] [8] [18]. In this regard, the "Mental Health in the UK and COVID-19" report indicated that increased depression, anxiety, and stress were associated with being younger and female during the pandemic [19].

The scores are in harmony with results of a nationwide survey of psychological distress among Chinese people in the COVID-19 where female respondents showed significantly higher psychological distress and more likely to develop post-traumatic stress disorder than their male counterparts [19]. Besides, Brazilian CPDI gender mean scores (41.1 and 33.2) in our research are higher than Chinese ones (24.87 and 21.41) [9].

Gender differences (higher levels of fear and distress among female respondents) could be interpreted as a social construction. It is possible that men, because not openly expressing their fears of the COVID-19, would not follow the preventive sanitary recommendations that the World Health Organization (WHO) uses to manage the spread of the coronavirus infection [20]: minimizing 
Table 2. Gender frequency of Peri-Traumatic Distress Scale (CPDI) and Fear scale (FCV-19S).

\begin{tabular}{|c|c|c|c|}
\hline & Women & Men & Sample \\
\hline \multicolumn{4}{|l|}{ CPDI statistics } \\
\hline Mean & 41.1 & 33.2 & 39.6 \\
\hline Median & 40 & 31 & 38 \\
\hline Mode & 43 & 29 & 30 \\
\hline Min & 4 & 8 & 4 \\
\hline Max & 93 & 85 & 93 \\
\hline Standard Deviation & 16.3 & 14.1 & 16.2 \\
\hline Standard Error & 0.4 & 0.7 & 0.4 \\
\hline \multicolumn{4}{|c|}{ CPDI Frequency of answers } \\
\hline Normal (0 - 28) & $375(25.5 \%)$ & $153(41.6 \%)$ & $530(28.7 \%)$ \\
\hline Mild (29 - 52) & $695(47.3 \%)$ & $178(48.4 \%)$ & $876(47.5 \%)$ \\
\hline Severe $(53-100)$ & $401(27.2 \%)$ & $37(10 \%)$ & $438(23.8 \%)$ \\
\hline \multicolumn{4}{|l|}{ FCV-19S statistics } \\
\hline Mean & 18.9 & 14.5 & 18.1 \\
\hline Median & 18 & 13 & 17 \\
\hline Mode & 16 & 12 & 16 \\
\hline Min & 7 & 7 & 7 \\
\hline Max & 35 & 35 & 35 \\
\hline Standard Deviation & 6.6 & 0.3 & 6.7 \\
\hline Standard Error & 0.2 & 5.9 & 0.2 \\
\hline \multicolumn{4}{|c|}{ FCV-19S Frequency of answers } \\
\hline Normal (7 - 16) & $590(40.1 \%)$ & $253(68.8 \%)$ & $844(45.8 \%)$ \\
\hline Mild (17 - 26) & $655(44.5 \%)$ & $97(26.4 \%)$ & $756(41 \%)$ \\
\hline Severe $(27-35)$ & $226(15.4 \%)$ & $18(4.8 \%)$ & $244(13.2 \%)$ \\
\hline
\end{tabular}

Note: The scores of CPDI range from 0 to 100; the scores of the FCV-19S range from 7 to 35.

the risk communication efforts of the authorities and media, not following hygiene practices, and social distancing-risk factors of the pandemic control.

CPDI scale shows that anxiety, exhaustion, and attention deficit were the factors with higher scores with important gender differences. Women showed higher levels of anxiety as they answered about feeling anxious during the pandemic as often (30.5\%) and most of the time (23.7\%). Men, on the contrary, showed lower levels of anxiety as they felt it often (21.2\%) and most of the time (13.6\%). A study with 2766 volunteers, [7] assessed anxiety during the pandemic. Results showed high levels as ranges varied from medium (81.3\%), high (7.2\%) and extremely high (11.5\%) associated with young age, female gender, family members infected with COVID-19, and a history of stressful situations and medical problems [7].

Considering that women frequently take on most of the burden and risk of Health care providers' roles at home, often with little external support [14], the 
fourteenth question of the scale that measures the exhaustion factor (I feel tired or even exhausted) showed big differences between genders: men had lower scores as they never $(20.7 \%)$ or occasionally (26.4\%) felt tired or even exhausted during the pandemic, while women felt that way often (22.5\%) and most of the time (27.9\%). Moreover, schools' closure has a differential effect on women, who provide most of the informal care within families [14].

The question of the scale, measuring the attention deficit factor, showed significant differences between genders: men's scores were lower as they never (20.9\%) or occasionally $(29.8 \%)$ found it hard to concentrate during the social isolation, whereas women's answers were often (19.9\%) and most of the time (24.8\%). These results could be associated with the higher levels of exhaustion showed by women during the pandemic and the cognitive differences between genders [13].

From the FCV-19S we could highlight two questions with significant gender differences on their answers: the first (I am most afraid of coronavirus-19), $74.1 \%$ of women agreed to be most afraid of coronavirus- 19 , while $34.8 \%$ of men selected those answers. The second question (It makes me uncomfortable to think about coronavirus-19), $66.2 \%$ of women agreed to felt uncomfortable thinking about the coronavirus-19, while $26.1 \%$ of men opted for those answers.

Figure 1 shows a strong, significant positive relationship between the FCV-19

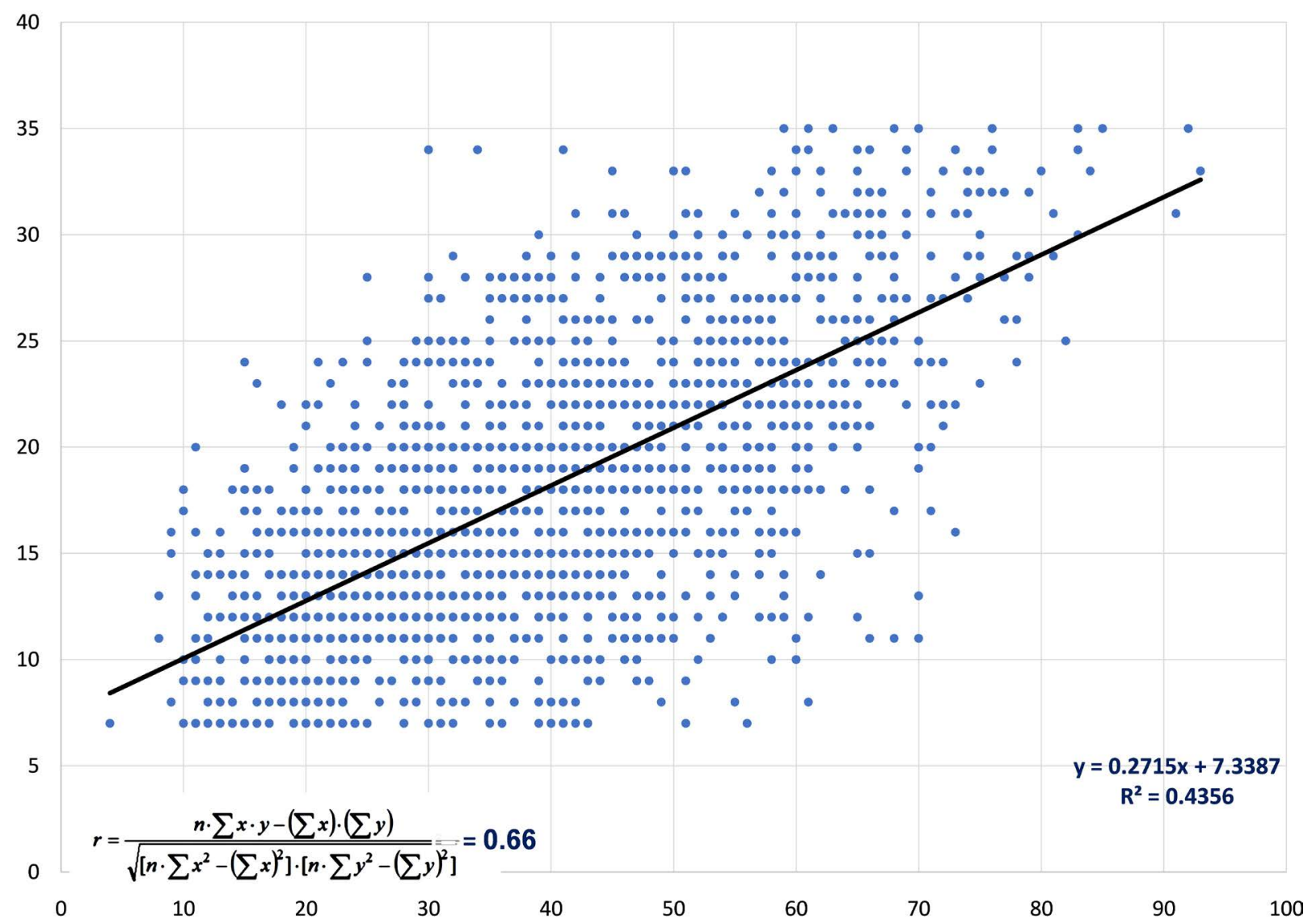

Figure 1. Peri-traumatic Distress Scale (CPDI) and Fear scale (FCV-19S) r Pearson coefficient using scatter diagram. 
Table 3. Cronbach's Alpha: Means and Confidence Interval CPDI and FCV-19S Scales.

\begin{tabular}{ccc}
\hline & CPDI Score & FCV-19S Score \\
\hline Minimum & 4 & 7 \\
Maximum & 93 & 35 \\
Median & 38 & 17 \\
Simple Average & 39.57 & 18.08 \\
Weighted Average & 39.57 & 1.99 \\
Confidence interval (95\%) & $38.83-40.31$ & $17.78-18.39$ \\
Standard Deviation & 16.24 & 6.69 \\
Cronbach's Alpha & 0.90 & 0.88 \\
\hline
\end{tabular}

scale and IEPC scale $(\mathrm{r}=0.660, \mathrm{p}<0.001)$. But the correlation between the FCV-19 scale and the S3 question of the IEPC scale (I feel terrified from imagining myself or my family being infected) shows a stronger correlation $(\mathrm{r}=$ $0.673, \mathrm{p}<0.001)$.

Internal consistencies on both scales are shown based on the Cronbach Alfa scores of CPDI and FCV-195 scales, being 0.90 and 0.88 respectively, obtained the greatest internal consistencies (Table 3). Additionally, the Kaiser-Meyer-Olkin (KMO) sample size suitability factor shows sample size adequacy with all CPDI values being $\geq 0.82$ except question 21 (0.57) and all FCV-195 Scale values being $\geq 0.84$.

At last, it is relevant to mention that this study has some limitations. First, although the participants were people for any region of Brazil, this survey should not be taken as a national sample; secondly, as most of the respondents had an incomplete or complete college degree, it does not reflect most of the Brazilian population.

\section{Conclusions}

CPDI and FCV-19S results show gender difference scores as a response to the pandemic. It is paramount that women's voices were represented in policy spaces as socially constructed gender roles place them in a strategic position to enhance multi-level interventions (primary and secondary effects of COVID-19), equitable policies, and new approaches to control the pandemic.

Finally, to control the viral spread in Brazil, it is of utmost importance to have a clear scientific-based pandemic control national guidelines (risk communication, hygiene, and social distance practices), the use of medications recommended by the World Health Organization (WHO) and health professionals on key positions in the Health Ministry.

\section{Authors' Contribution}

A. Abad, L.E.P.P. Teixeira, Juliana A. da Silva and R.L. de Freitas elaborated, analysed, and wrote sed the manuscript; José A. da Silva participated in the 
created, elaborated the project and revised the manuscript; S. Paiva, S. Bastos, C.H.C. Mármora, L.A.M. Campos and M. Antonelli-Ponti, revised the manuscript.

\section{Conflicts of Interest}

The authors declare no conflicts of interest regarding the publication of this paper.

\section{References}

[1] Wang, D., Hu, B., Hu, C., Zhu, F., Liu, X., Zhang, J., et al. (2020) Clinical Characteristics of 138 Hospitalized Patients with 2019 Novel Coronavirus-Infected Pneumonia in Wuhan, China. JAMA, 323, 1061-1069. https://doi.org/10.1001/jama.2020.1585

[2] Abad, A., Da Silva, J., Das Neves Braga, J., Medeiros, P., De Freitas, R., Coimbra, N. and Da Silva, J. (2020) Preparing For the COVID-19 Mental Health Crisis in Latin America-Using Early Evidence from Countries That Experienced COVID-19 First. Advances in Infectious Diseases, 10, 40-44. https://doi.org/10.4236/aid.2020.103005

[3] Choi, E.P.H., Hui, B.P.H. and Wan, E.Y.F. (2020) Depression and Anxiety in Hong Kong during COVID-19. International Journal of Environmental Research and Public Health, 17, 3740. https://doi.org/10.3390/ijerph17103740

[4] Taylor, S. (2019) The Psychology of Pandemics: Preparing For the Next Global Outbreak of Infectious Disease. Cambridge Scholars Publishing, Cambridge.

[5] Danese, S., Cecconi, M. and Spinelli, A. (2020) Management of IBD during the COVID-19 Outbreak: Resetting Clinical Priorities. Nature Reviews Gastroenterology and Hepatology, 17, 253-255. https://doi.org/10.1038/s41575-020-0294-8

[6] Jahanshahi, A.A., Dinani, M.M., Madavani, A.N., Li, J. and Zhang, S.X. (2020) The Distress of Iranian Adults during the Covid-19 Pandemic-More Distressed than the Chinese and with Different Predictors. Brain, Behavior, and Immunity, 87, 124-125 https://doi.org/10.1016/j.bbi.2020.04.081

[7] Mazza, C., Ricci, E., Biondi, S., Colasanti, M., Ferracuti, S., Napoli, C. and Roma, P. (2020) A Nationwide Survey of Psychological Distress among Italian People during the COVID-19 Pandemic: Immediate Psychological Responses and Associated Factors. International Journal of Environmental Research and Public Health, 17, 3165. https://doi.org/10.3390/ijerph17093165

[8] Qiu, J., Shen, B., Zhao, M., Wang, Z., Xie, B. and Xu, Y. (2020) A Nationwide Survey of Psychological Distress among Chinese People in the COVID-19 Epidemic: Implications and Policy Recommendations. General Psychiatry, 33, e100213. https://doi.org/10.1136/gpsych-2020-100213

[9] Ahorsu, D.K., Lin, C.Y., Imani, V., Saffari, M., Griffiths, M.D. and Pakpour, A.H. (2020) The Fear of COVID-19 Scale: Development and Initial Validation. International Journal of Mental Health and Addiction. https://doi.org/10.1007/s11469-020-00270-8

[10] Bao, Y.P., Sun, Y.K., Meng, S.Q., Shi, J. and Lu, L. (2020) Covid-19 Epidemic: Address Mental Health Care to Empower Society. The Lancet, 395, E37-E38. https://doi.org/10.1016/S0140-6736(20)30309-3

[11] Veer, I.M., Riepenhausen, A., Zerban, M., Wackerhagen, C., Engen, H., Puhlmann, L., et al. (2020) Mental Resilience in the Corona Lockdown: First Empirical Insights from Europe. 
[12] Fernandes, H. (2020) The Main Risk Factors For the Number of Serious or Critical Cases of Covid-19: How Is the Health of Brazilians? Preprints, 2020050143. https://doi.org/10.20944/preprints202005.0143.v1

[13] Regitz-Zagrosek, V. (2012) Sex and Gender Differences in Health. EMBO Reports, 13, 596-603. https://doi.org/10.1038/embor.2012.87

[14] Smith J. (2019) Overcoming the "Tyranny of the Urgent": Integrating Gender into Disease Outbreak Preparedness and Response. Gender and Development, 27, 355-369. https://doi.org/10.1080/13552074.2019.1615288

[15] Rugema, L., Persson, M., Mogren, I., Ntaganira, J. and Krantz, G. (2019) A Qualitative Study of Healthcare Professionals' Perceptions of Men and Women's Mental Healthcare Seeking in Rwanda. Journal of Community Psychology, 48, 891-903. https://doi.org/10.1002/jcop.22308

[16] Lauri Korajlija, A. and Jokic-Begic, N. (2020) COVID-19: Concerns and Behaviours in Croatia. British Journal of Health Psychology. https://doi.org/10.1111/bjhp.12425

[17] Wenham, C., Smith, J. and Morgan, R. (2020) COVID-19: The Gendered Impacts of the Outbreak. The Lancet, 395, 846-848. https://doi.org/10.1016/S0140-6736(20)30526-2

[18] Olagoke, A.A., Olagoke, O.O. and Hughes, A.M. (2020) Exposure to Coronavirus News on Mainstream Media: the Role of Risk Perceptions and Depression. British Journal of Health Psychology, e12427. https://doi.org/10.1111/bjhp.12427

[19] Jia, R., Ayling, K., Chalder, T., Massey, A., Broadbent, E., Coupland, C. and Vedhara, K. (2020) Mental Health in the UK during the COVID-19 Pandemic: Early Observations. https://doi.org/10.1101/2020.05.14.20102012

[20] World Health Organization (2008) WHO Outbreak Communication Planning Guide. World Health Organization, Geneva. 